\title{
Malignant Lymphoma in the Mesentery with Immune Thrombocytopenia
}

\author{
Yuji Moriwaki, Michiko Naka, Tetsuya Yamamoto, Shotaro Takagi, Toshihiro Hidaka, \\ Sumio Takahashi, Toshikazu Hada, Takashi Nishigami* and Kazuya Higashino
}

\begin{abstract}
A 69-year-old man was referred to our hospital for further evaluation of an abdominal mass. After admission, he was suspected of having a malignant mesenteric tumor. Laboratory data disclosed thrombocytopenia with increased levels of platelet-associated immunoglobulin G. On surgery, the tumor involved the ileal mesentery, invading the urinary bladder and mucosal surface of the terminal ileum. The diagnosis of mesenteric lymphoma with immune thrombocytopenia was made. Complete remission was obtained after surgery and the subsequent three courses of combination chemotherapy. However, thrombocytopenia still persisted. This rare presentation is discussed with a review of the available literature.
\end{abstract}

(Internal Medicine 31: 1185-1189, 1992)

Key words: abdominal lymphoma, mesenteric tumor, immune thrombocytopenic purpura, platelet-associated immunoglobulin $\mathrm{G}$ (PA-IgG)

\section{Introduction}

Malignant lymphoma refers to a general term for non-epithelial malignant tumor originating from lymphatic tissues; it rarely occurs in the mesentery.

Immune thrombocytopenia may occur in lymphoproliferative diseases such as chronic lymphocytic leukemia (1), but it is distinctly rare in patients with malignant lymphoma. In this paper, we present a patient with non-Hodgkin malignant lymphoma in the mesentery who also displayed immune thrombocytopenia. The implication of the relationship between malignant lymphoma and immune thrombocytopenia is discussed with a review of the available literature.

\section{Case Report}

A 69-year-old man was referred to our hospital for further evaluation of a palpable right lower quadrant abdominal mass on August 28, 1990. He had undergone an operation for aneurysm of the abdominal aorta 8 years earlier, at which time the thrombocyte number was normal, ranging from $12.5-27.3 \times 10^{4} / \mathrm{mm}^{3}$. On admission, blood pressure was $140 / 94 \mathrm{mmHg}$. No superficial lymph nodes were palpable. There was no petechial purpura over the skin. The lungs and heart were normal. A solid mass, measuring $8 \times 6 \mathrm{~cm}$, was palpated in the lower right quadrant of the abdomen. However, the liver, spleen and kidneys were not palpable. Laboratory studies disclosed the following: The red blood cell count was $467 \times 10^{4} / \mathrm{mm}^{3}$, hematocrit $40.1 \%$ and hemoglobin $12.9 \mathrm{~g} / \mathrm{dl}$. The white blood cell count was $14,800 / \mathrm{mm}^{3}$ with $37 \%$ polymorphonuclear leukocytes, $18 \%$ lymphocytes and $44 \%$ monocytes. The platelet count was $5.6 \times$ $10^{4} / \mathrm{mm}^{3}$. Prothrombin time and plasma fibrinogen were normal. The blood sedimentation rate was $9 \mathrm{~mm} /$ hour and C-reactive protein was $2.4 \mathrm{mg} / \mathrm{dl}$. The serum total protein was $7.6 \mathrm{~g} / \mathrm{dl}$ with an albumin concentration of $4.0 \mathrm{~g} / \mathrm{dl}$. Renal and hepatic function tests as well as serum electrolytes were normal. Serum immunoglobulin levels were normal. Platelet-associated immunoglobulin G (PA-IgG) was positive with a maximum level of $183.7 \mathrm{ng} / 10^{7}$ platelet (normal $<25 \mathrm{ng} / 10^{7}$ platelet). Coombs' test was negative. The urine was normal. Fecal occult blood examination gave positive results on several occasions. The bone marrow aspirate revealed normocellular marrow (nucleated cell count $7.5 \times 10^{4} / \mathrm{mm}^{3}$ ) with adequate number $\left(46.8-62.4 / \mathrm{mm}^{3}\right)$ of normalappearing megakaryocytes and showed no evidence of invasion of malignant lymphoma cells. The levels of

From the Third Department of Internal Medicine, Hyogo College of Medicine and *the Second Department of Pathology, Hyogo College of Medicine, Hyogo

Received for publication November 7, 1991; Accepted for publication July 15, 1992

Reprint requests should be addressed to Dr. Yuji Moriwaki, the Third Department of Internal Medicine, Hyogo College of Medicine, Mukogawacho 1-1, Nishinomiya, Hyogo 663, Japan 
M-CSF (colony stimulating factor) and GM-CSF were below the detection limit. Terminal ileum and a part of ascending colon were displaced by the mass, but the mucosal surface and wall of the intestine were smooth on barium enema study (Fig. 1). Ultrasonography revealed an oblong, slightly lobulated sonolucent solid mass, measuring $9 \times 6 \times 7 \mathrm{~cm}$ with a central area of increased echogenecity (Fig. 2). A CT scan demonstrated a plaquelike, solid mass enveloping the mesenteric fat (Fig. 3). There was no evidence of periaortic or retroperitoneal adenopathy. The result of drip infusion pyelography (DIP) was not remarkable. On superior mesenteric arteriography, the tumor was hypovascular and the arteries were distended with smooth encasement in the periphery (Fig. 4). A ${ }^{67} \mathrm{Ga}$ citrate radionuclide scan showed the uptake of gallium citrate in the lower midabdomen, coincident with the site of the tumor (Fig. 5). Foot lymphography gave normal results.

On surgery, the tumor was located in the mesentery surrounded by the cecum and terminal ileum, measuring $13 \times 10 \mathrm{~cm}$; part of the tumor invaded the urinary bladder and mucosal surface of the terminal ileum, forming an ulcer (Fig. 6). The tumor was resected completely with partial ileotomy. Splenectomy was not performed because the spleen appeared normal. The cut surface of the resected specimen was mostly white, and partly

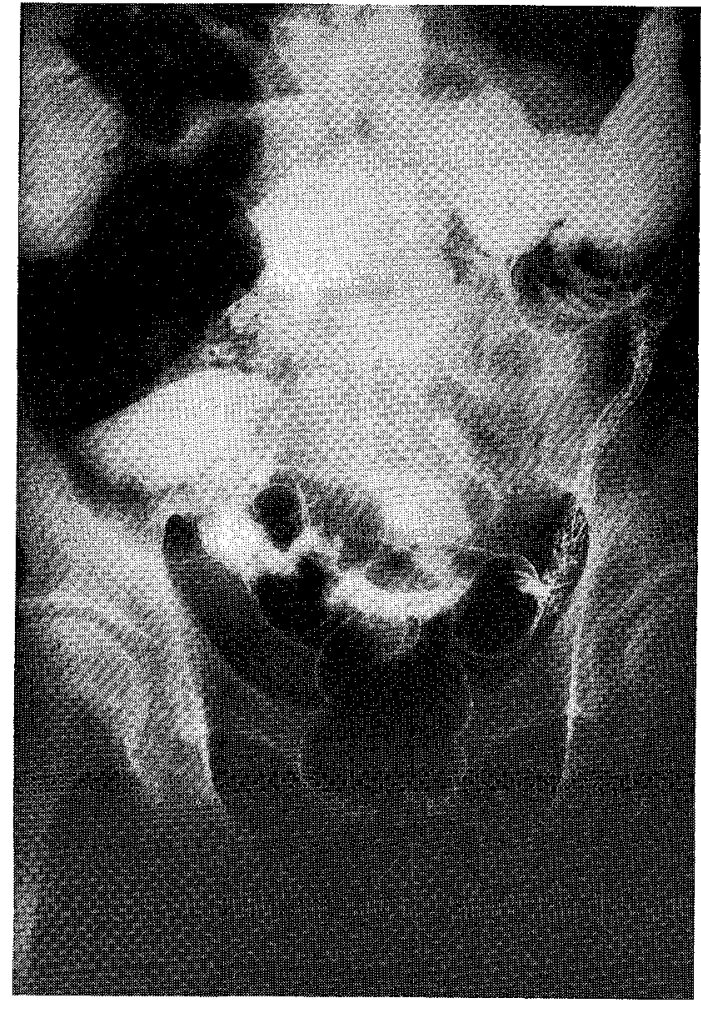

Fig. 1. Barium cnema showing displacement of the terminal ileum and a part of the ascending colon by the tumor. yellowish with hemorrhagic spots. Microscopically, the tumor cells with a large nucleus proliferated invasively,

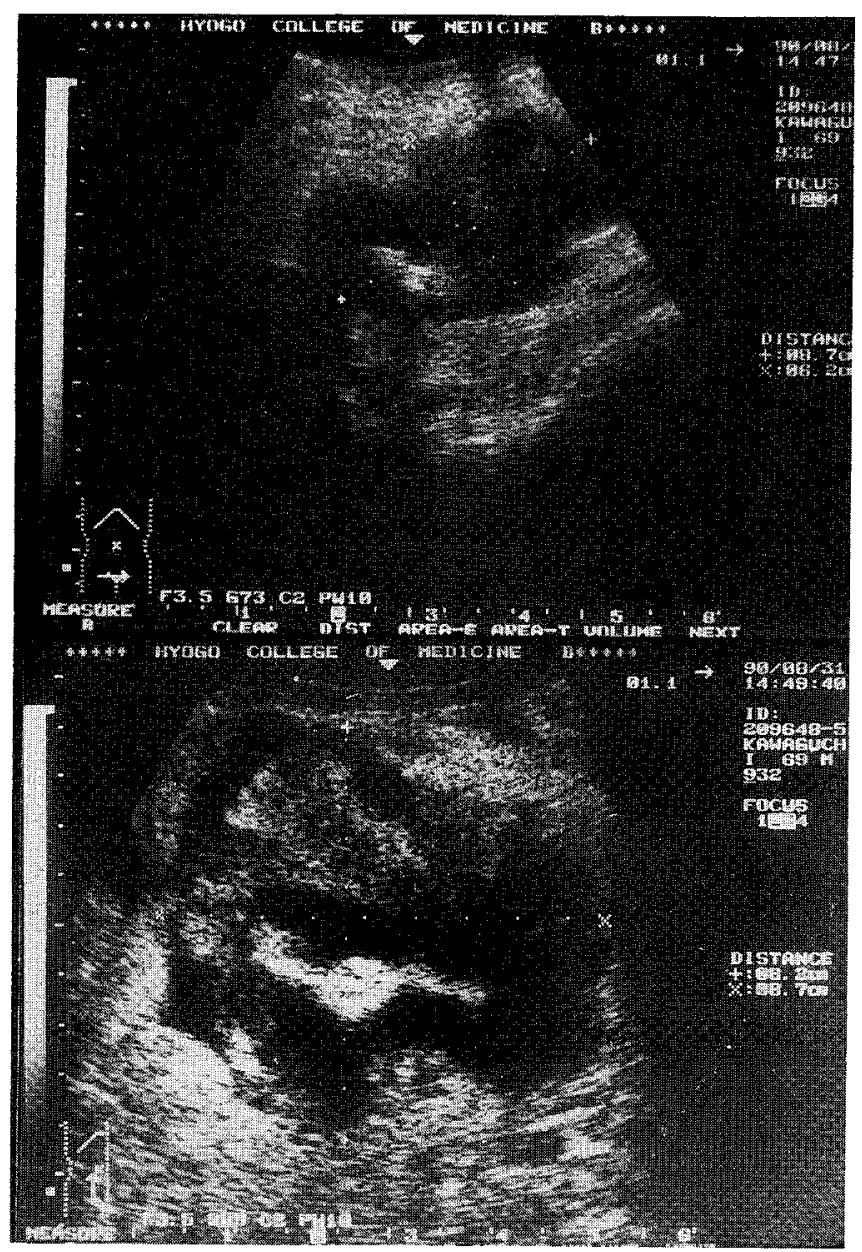

Fig. 2. Ultrasonography of the abdomen showing an oblong, slightly lobulated sonolucent solid mass with a central area of increased echogenecity.

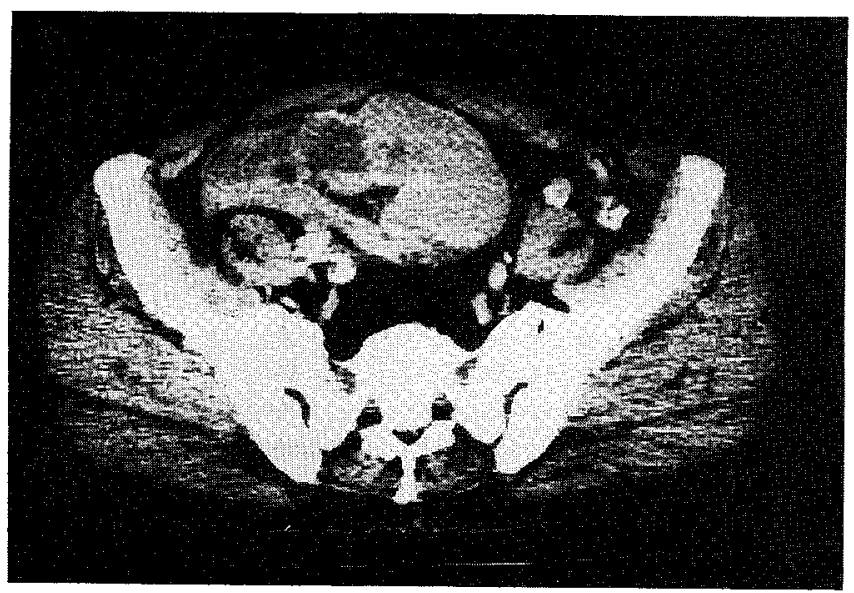

Fig. 3. CT scan of the abdomen demonstrating a plaque-like, solid mass enveloping the mesenteric fat. 


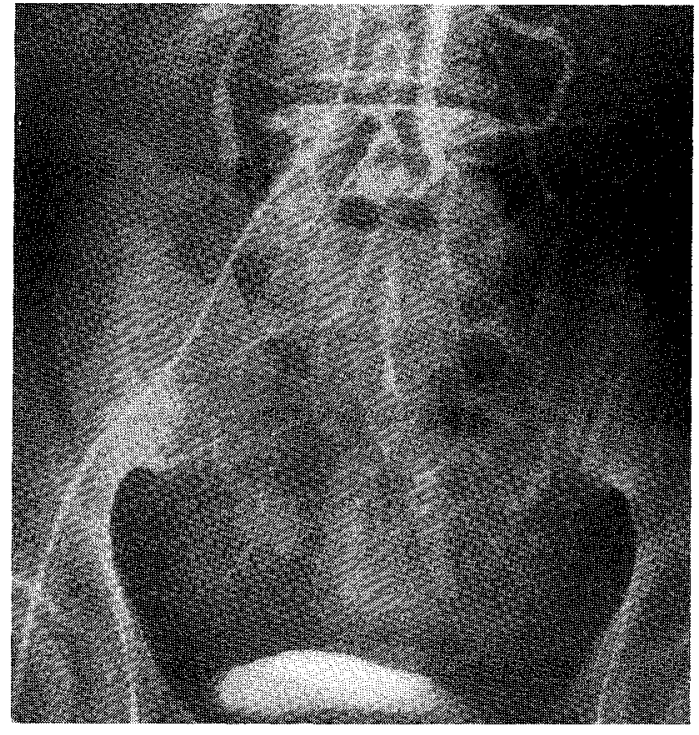

Fig. 4. The tumor was hypovascular and the arteries were distended with smooth encasement in the periphery on angiography.

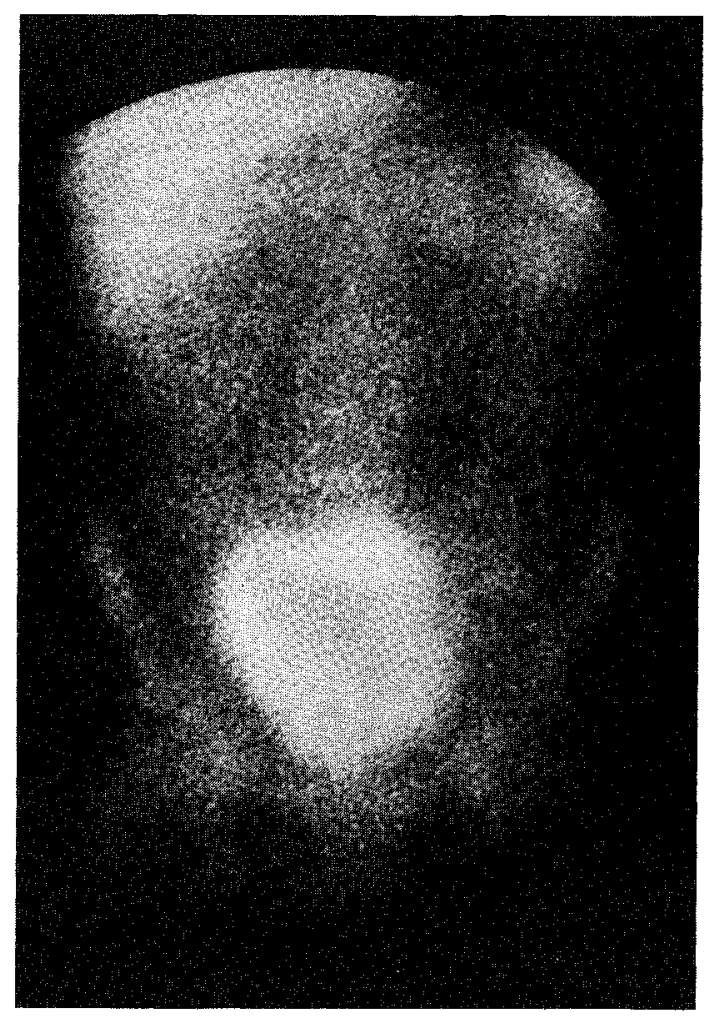

Fig. 5. ${ }^{67}$ Gallium radionuclide scan disclosing an accumulation of ${ }^{67}$ gallium citrate in the lower mid abdomen.

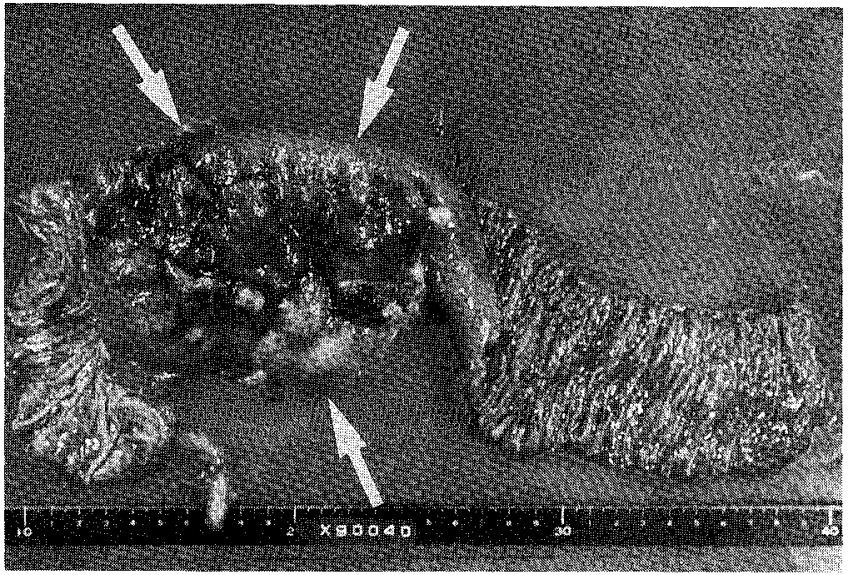

Fig. 6. Resected specimen of the tumor; the tumor was located in the mesentery surrounded by the cecum and terminal ileum.

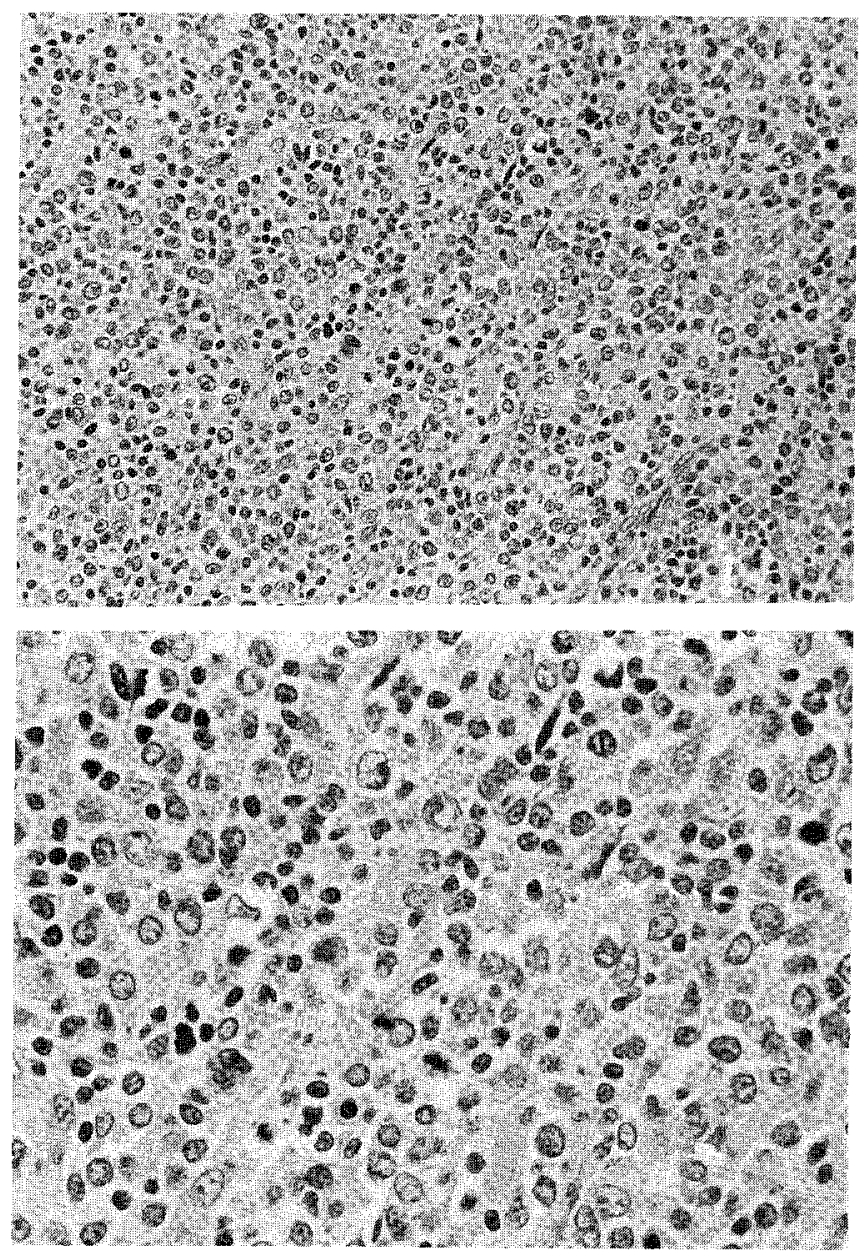

Fig. 7. Microscopically, the tumor cells with a large nucleus were proliferated invasively, destroying normal follicular structure. Mitotic figures are also observed (HE stain, $\times 100, \times 200$ ). 
destroying the normal follicular structure (Fig. 7). Immunohistochemical staining using antihuman $B$ cell mouse monoclonal antibody (Dako-L26) was positive, while that for T cells (Dako-UCHL1) was negative. The histopathological diagnosis of non-Hodgkin lymphoma (diffuse large, B cell type, clinical stage IV) in the mesentery was made. After surgery, three courses of combination chemotherapy (cyclophosphamide, epirubicin, vincristine and prednisolone) were instituted and clinical remission was achieved. However, thrombocytopenia still persisted.

\section{Discussion}

Primary mesenteric tumor is generally considered rare and its occurrence has been only about $0.00018 \%$ $(8 / 444,332)$ to $0.012 \%(6 / 50,000)$ of inpatients in the series reported by Steinreich (2) and Majnarich (3). According to Katsuda et al (4), malignant lymphoma accounted for $6.5 \%$ of 107 cases of mesenteric tumors documented in Japan from 1965 to 1975 . In another study, malignant lymphoma arising from the mesentery occupied only 12 out of 648 cases $(2 \%)$ of malignant lymphomas from 1955 to 1979 (5). Malignant lymphoma arising from the mesentery is, therefore, considered very rare.

Palpable mass and/or abdominal pain are the most common symptoms present in mesenteric tumors, while leukemic change, fever, weight loss and cachexia are present in some advanced cases of mesenteric malignant lymphoma as generalized manifestations. However, mesenteric lymphomas are usually insidious and they may attain a large size before producing symptoms as commonly associated with other mesenteric tumors.
The diagnosis of mesenteric lymphoma is often difficult, but with the development of CT scan and ultrasonography, the detection of such tumors is expected to increase. The encasement of the superior mesenteric artery and fat tissue by tumor infiltrating the mesenteric leaves on CT scan or ultrasonography is a characteristic appearance in mesenteric lymphoma and it is often called the "sandwich sign" (6). In the present case, the encasement of fat tissue by the tumor was identified. However, the superior mesenteric artery was not clearly demonstrated within it. On angiography, mesenteric lymphoma often appears either hypovascular (as with the present case) or avascular, but may sometimes be hypervascular with diffuse staining during the capillary phase (7).

Another characteristic feature of the present case is thrombocytopenia. Thrombocytopenia is a common complication of advanced lymphoproliferative disease. It is often presumed to be due to marrow infiltration or chemo-radiotherapy and less commonly due to hypersplenism, $(8-10)$ while it may be ascribed to the disturbances of immune function present in patients with malignant lymphoma (11). These patients often present with several autoantibodies such as to red cells, neutrophils and to thrombocytes. The present case had an adequate number of megakaryocytes and no evidence of tumor cell infiltration in the bone marrow, small spleen, or other illnesses associated with immune thrombocytopenia. Further, the thrombocyte count was inversely correlated with the level of PA-IgG $(r=-0.97$, $\mathrm{p}<0.01, \mathrm{n}=6$ ) (Fig. 8, inset) in addition to the presence of thrombocytopenia before chemotherapy. These findings support the notion that immune destruction due to an elevated level of PA-IgG could be the cause of

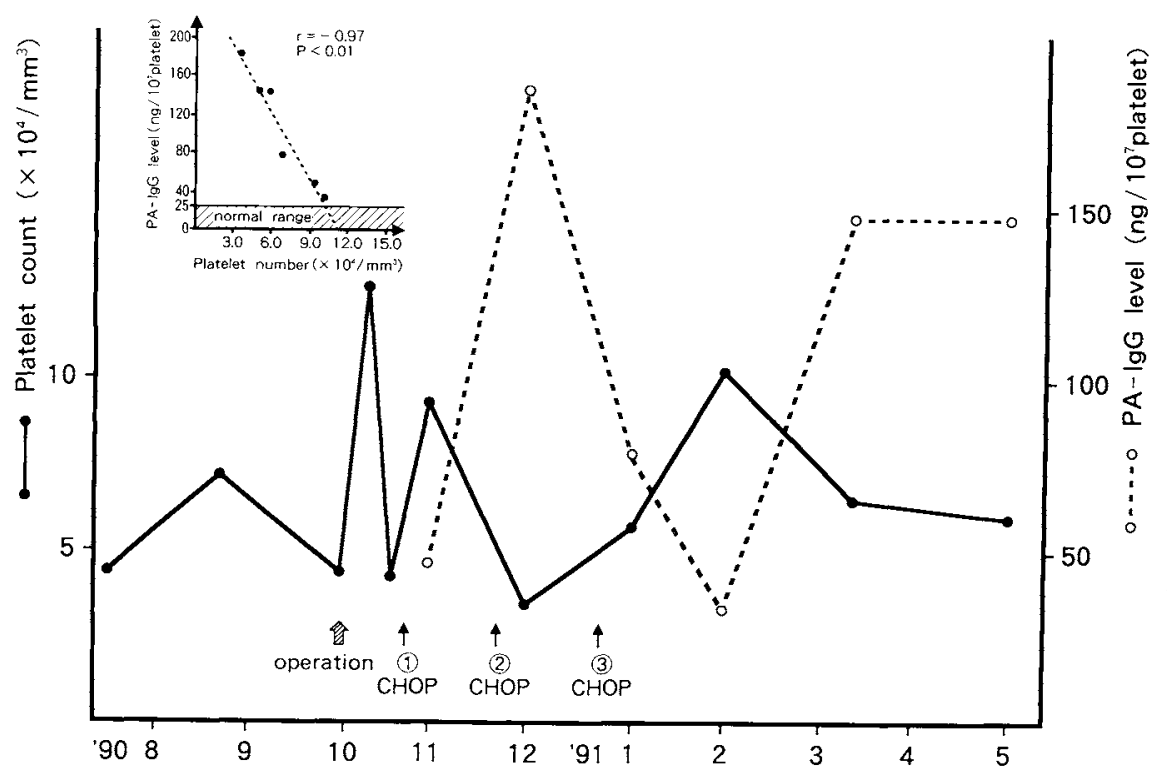

Fig. 8. Clinical course and correlation between thrombocyte count and PA-IgG level. 


\section{Mesenteric Lymphoma, Immune Thrombocytopenia}

thrombocytopenia in the present case.

According to Berkman et al (12), PA-IgG is usually elevated in patients with lymphoma (39\% in Hodgkin's disease and $20 \%$ in non-Hodgkin's lymphoma), but its concentration is not always related to the presence of thrombocytopenia. Immune thrombocytopenic purpura was observed only in $1 \%(13)$ and $0.4 \%$ (14) in a large series of patients with Hodgkin's disease and non-Hodgkin lymphoma, respectively. Therefore, the incidence of immune thrombocytopenia is distinctly rare in patients with malignant lymphoma.

Fink and Al-Mondhiry (15) reviewed 17 patients with idiopathic thrombocytopenic purpura (ITP) associated with malignant lymphoma and reported that ITP was coincidental with the diagnosis of malignant lymphoma in $6(35 \%)$, but in 9 patients $(53 \%)$, ITP developed several months after the diagnosis of malignant lymphoma had been established, while in the remaining $2(12 \%)$, ITP heralded the onset of lymphoma. In the present case, the thrombocyte count was normal 8 years previously, but the thrombocyte count just prior to the onset of lymphoma was not available and therefore, we could not determine the exact relationship between the emergence of immune thrombocytopenia and malignant lymphoma.

It is reported that ITP may be encountered in all stages and histological subtypes of Hodgkin's disease (13). In contrast, the extent of non-Hodgkin lymphoma by stage and histopathologic type at the time of immune thrombocytopenia has not been previously explored in detail.

It is known that herpes zoster or other viral infections may induce immune thrombocytopenic purpura (13) and the possibility of cytomegalovirus inducing thrombocytopenia in Hodgkin's disease has been discussed (16). However, in the present case, there was no definite evidence suggestive of such viral infections.

The occurrence of immune thrombocytopenic purpura seems to correlate with the activity of the underlying lymphoma (15) and it has also been reported that PAIgG may be a useful marker for disease activity in patients with advanced Hodgkin's disease (16), suggesting that PA-IgG is produced by the tumor. In the present case, the origin of PA-IgG and the significance of PA-IgG as a disease marker are not clear, especially considering the fact that normalization of the thrombocyte count and PA-IgG level did not occur in spite of the successful control of the underlying lymphoma by operation and subsequent chemotherapy (Fig. 8).

In conclusion, the association between immune thrombocytopenia and malignant lymphoma (especially non-Hodgkin type) is unclear in many aspects and remains to be clarified by further study with more extensive cases including the present case.

This casc was presented at the 135th Annual Meeting of Internal Medicine of Kinki District held in Kyoto on Sept. 14, 1991.

Acknowledgement: The authors are grateful to Dr. Masaki Fujiwara and Dr. Teruyuki Kuroki (Second Department of Surgery) for their surgical cooperation.

\section{References}

1) Kaden BR, Rosse WF, Hauch TW. Immune thrombocytopenia in lymphoproliferative discases. Blood 53: 545, 1979.

2) Steinreich OS. The diagnosis of mesenteric cysts. Ann Surg 142: 889,1955

3) Majnarich G. Mesenteric, mesocolic and omental tumors with particular reference to cystic forms. J Inter Coll Surg 24: 403, 1955.

4) Katsuda $\mathrm{H}$, Arimoto $\mathrm{S}$, Naito $\mathrm{Y}$, et al. A case of mesenteric malignant lymphoma. Geka-Shinryo 359: 101, 1978 (in Japanese).

5) Shirakawa S, Tatsumi E, Nasu K, Kita K, Uchino H. Clinical pictures of malignant lymphoma in the gastrointestinal tractProgression of disease and prognosis. Recent Advances in RES Research 20: 61, 1981.

6) Mueller PR, Ferrucci JT, Harbin WP, Kirkpatrick RH, Simeone JF, Wittenberg J. Appearance of lymphomatous involvement of the mesentery by ultrasonography and body computed tomography: the "sandwich sign". Radiology 134: 467, 1980.

7) Levin DC, Watson RC, Baltaxc HA. Arteriography of retroperitoneal masses. Radiology 108: 543, 1973.

8) Cohen JR. Idiopathic thrombocytopenic purpura in Hodgkin's disease. A rare occurrence of no prognostic significance. Cancer 41: 743, 1978.

9) Jalia A, Miller SP. Idiopathic thrombocytopenic purpura in Hodgkin's discase after splenectomy. Am $\int$ Hematol 1: 115, 1976.

10) Kedar A, Khan AB, Mattern JQ, Fisher J, Thomas PRM, Freeman AI. Autoimmune disorders complicating adolescent Hodgkin's disease. Cancer 44: 112, 1979.

11) Twomey JJ, Rice L. Impact of Hodgkin's disease upon the immune system. Semin Oncol 7: 114, 1980

12) Berkman AW, Kickler T, Braine H. Platelet-associated IgG in patients with lymphoma. Blood 63: 944, 1984.

13) Xiros N, Binder T, Anger B, Bohlke J, Heimpel H. Idiopathic thrombocytopenic purpura and autoimmune hemolytic anemia in Hodgkin's disease. Eur J Haematol 40: 437, 1988.

14) Jones SE. Autoimmune disorders and malignant lymphoma. Cancer 31: 1092, 1973.

15) Fink K, Al-Mondhiry H. Idiopathic thrombocytopenic purpura in lymphoma. Cancer 37: 1999, 1976.

16) Duhamel G, Moise A, Najman A, et al. Maladie de Hodgkin, purpura thrombopénique, polyradiculonévrite et pneumopathie à cytomégalovirus. Ann Méd Interne 130: 449, 1979 (in French). 